\title{
Feeling the shape: active exploration behaviours for object recognition with a robotic hand
}

\author{
Uriel Martinez-Hernandez, Tony J. Dodd and Tony J. Prescott
}

\begin{abstract}
Autonomous exploration in robotics is a crucial feature to achieve robust and safe systems capable to interact with and recognise their surrounding environment. In this work, we present a method for object recognition using a three-fingered robotic hand actively exploring interesting object locations to reduce uncertainty. We present a novel probabilistic perception approach with a Bayesian formulation to iteratively accumulate evidence from robot touch. Exploration of better locations for perception is performed by familiarity and novelty exploration behaviours, which intelligently control the robot hand to move towards locations with low and high levels of interestingness respectively. These are active behaviours that, similar to the exploratory procedures observed in humans, allow robots to autonomously explore locations they believe that contain interesting information for recognition. Active behaviours are validated with object recognition experiments in both off-line and realtime modes. Furthermore, the effects of inhibiting the active behaviours are analysed with a passive exploration strategy. The results from the experiments demonstrate the accuracy of our proposed methods, but also their benefits for active robot control to intelligently explore and interact with the environment.
\end{abstract}

Index Terms-Active exploration, Bayesian perception, intrinsic motivation and shape recognition.

\section{INTRODUCTION}

$\mathbf{T}$ OUCHING and feeling are processes that allow intelligent autonomous robots to understand and interact with their surrounding environment. Even though these are easy tasks performed day to day by humans, they represent complex processes for autonomous robots. Advances in technology have shown great progress in the development of touch sensors that mimic receptors and functionalities of human hands and fingers for multiple applications [1], [2], [3]. However, humans not only touch but also feel, purposefully moving their hands and fingers through exploratory procedures to enhance the perceptual characteristics of what is being touched [4], [5]. For that reason, investigation on computational algorithms that allow autonomous robots to explore, perceive and feel what they are touching is essential to understand the state of their surrounding environment.

Manuscript submitted on August, 2016. Resubmitted on January, 2017. Resubmitted on April, 2017. This work was supported by EU Framework WYSIWYD (FP7-ICT-2013-10).

U. Martinez-Hernandez is with the Institute of Design, Robotics and Optimisation (iDRO) and the School of Mechanical Engineering, The University of Leeds, Leeds, LS2 9JT, UK (email: u.martinez@leeds.ac.uk)

Tony J. Dodd is with the Sheffield Robotics Laboratory and the Department of Automatic Control and Systems Engineering, The University of Sheffield, Mappin Street, Sheffield, S1 3JD, UK (email: t.j.dodd@sheffield.ac.uk)

Tony J. Prescott is with the Sheffield Robotics Laboratory and the Department of Psychology, The University of Sheffield, Mappin Street, Sheffield, S1 3JD, UK (email: t.j.prescott@sheffield.ac.uk)
In this work, we present a novel approach for object exploration and recognition, that allows a robotic hand to autonomously inspect interesting object locations that provide better information to improve perception. Our approach is composed of a Bayesian formulation for perception of touch and two active exploration strategies. For perception of robot touch, we use a probabilistic Bayesian formulation which has shown to be accurate for perception using a fingertip sensor and various stimuli [6], [7], [8]. This probabilistic formulation, together with a sequential analysis method, allows robots to autonomously accumulate evidence and make decisions about the objects being explored. Furthermore, probabilistic approaches offer to robotics not only a robust platform to deal with sensor noise and environment dynamics, but also to handle the uncertainty, present in the measurements, to act accordingly in unstructured environments [9], [10].

We investigate active strategies to purposefully move a robotic hand for exploration of object locations that contain low and high levels of interestingness. We propose the integration of our perception method with two active exploration methods; familiarity and novelty behaviours. They allow to intelligently control robot movements, seeking better object locations that improve perception during an exploration task. These methods extend our previous study on active exploration of object shape [11]. The familiarity and novelty behaviours are inspired by intrinsic motivation research in psychology and neuroscience, which has been demonstrated to be primordial for cognitive development and engagement of humans to explore and manipulate their environment [12], [13], [14]. Also, our methods mimic the observed results from investigations on perception, which have shown that humans not only touch, but also feel by actively exploring their environment [15], [16].

Validation of our methods is performed with exploration and recognition of object shape experiments in off-line and realtime modes. For this process, multiple datasets composed of touch and proprioceptive data are collected from real objects for training and testing. In off-line mode validation, multiple testing datasets are constructed with real data. For real-time validation, a three-fingered robotic hand and a positioning robot are employed. We also analyse the effects on the object exploration task, when the robot movements are not controlled by sensory feedback. The results demonstrate that knowing where to explore to reduce uncertainty, improves the perception accuracy over a random exploration behaviour. Furthermore, we observe that the exploration of object locations with high levels of interestingness, identified by the novelty exploration behaviour, allows the robot hand to achieve a better trade-off between perception accuracy and reaction time. 
Overall, our work provides a novel approach that, composed of a Bayesian formulation and two active exploration behaviours based on intrinsic motivation models, offers multiple benefits to develop autonomous robots capable to mimic the human behaviour for tactile exploration tasks.

This paper is organised as follows: First, a description of related work is presented in Section II. Second, our proposed methods for tactile exploration and recognition are described in Section III. Third, experiments and results are presented in Section IV. Fourth, Section V presents the discussion of our work. Finally, conclusions are presented in Section VI.

\section{RELATED WORK}

Exploratory procedures employed by humans using their hands and fingers have inspired the investigation of methods for perception and exploration in robotics. One of the first tactile robot systems, built with one finger sensor, proposed a basic set of features required for object recognition, for instance, compliance, texture, edges, contours and corners [17], [18]. Identification of these tactile features has been studied using different approaches. Model-based and template matching methods have been used for object recognition from multiple sensory inputs, e.g., touch and vision [19], [20]. Normally, these methods directly compare an input dataset with a codebook, and without using an uncertainty measure or selection of relevant features, which reduces the accuracy, speed and reliability of the recognition task. A neural network, trained with tactile images from a robotic gripper, allowed the recognition in off-line mode of a limited number of objects [21]. Tactile and force features were used for object exploration using rolling and sliding processes [22]. However, these exploration methods were limited to a fixed number of steps without adaptability for exploration of new object shapes. Other computational methods such as principal component analysis (PCA), image moments and self-organising maps (SOM) allowed to recognise objects using a predefined and fixed sequence of exploration contacts with low recognition accuracy [23], [24]. The main drawback of previous works is their lack of perception and decision methods, which play a key role to achieve autonomous robots that intelligently explore and interact with their environment.

A study on geometric constraints showed how a robot arm was capable to choose the set of exploration movements to recognise an object [25]. However, this work was limited to fully known object models. An approach based on the generation of curves, that represent the optimal path for object exploration, also required full knowledge of the object [26]. Tactile images and haptic information have been widely used with multi-layer neural networks to explore and recognise object shape [27], [28], [29]. Unfortunately, these works are bounded by prior knowledge of object geometry and size, as well as by black box models generated by neural networks. Probabilistic methods have also been studied in multiple robotic applications, offering a robust framework for learning, perception, control and interaction [30], [31]. Probabilistic representation of tactile data and point clouds allowed the recognition of household objects in off-line mode using a fixed sequence of contact locations for exploration [32], [33]. The bag-of-features approach has been employed in different scenarios for tactile perception and identification, e.g., object identification with a tactile gripper [34]. However, this method requires the exploration of the complete object by the gripper, rather than autonomously deciding where to explore to improve both, the time to finish the task and the computational cost. Accurate touch and object recognition were achieved using Gaussian Processes (GP) and Deep Learning techniques with a robot interacting with humans and their environment [35], [36]. Despite the high accuracy achieved, this method required a fixed sequence of tactile contacts, with no autonomous decision for exploration of better object locations to reduce uncertainty as humans do. Active sensing, together with Bayesian formulations, have allowed a biomimetic fingertip sensor to explore better locations for perception, tracing and extracting object shapes in off-line and real-time modes [37], [38]. An active sensing method with a probabilistic approach allowed a vision system to look to specific areas in the visual scene with higher saliency [39]. Research on cognitive robotics has shown that perception methods, together with intrinsic motivation models, have the potential to develop robots that intelligently explore their environment seeking interesting information that allow them to improve their knowledge [40], [41].

An intrinsically motivated robot, integrated with a novelty model and rewards, using vision and touch sensing, learned to observe towards salient or interesting stimuli while neglecting unimportant inputs [42]. Cognitive architectures for active exploration, learning, information-seeking and attention with computational agents, were proposed with multiple intrinsic motivation models, e.g., information gain, predictive novelty, distributional surprise, distributional familiarity [43], [44], [45]. Learning of robot skills was investigated with intrinsically motivated movements, allowing a robot to explore interesting stimuli while maximising visual and tactile perception [46], [47]. Active exploration and intrinsic motivation allowed a robot to learn inverse models and motor primitives [48], [49]. This work also showed the benefits of actively selecting goals, based on maximal improvement, rather than following a random selection or passive approach.

We propose a novel method for object recognition through the intelligent exploration of interesting locations that permit to improve tactile perception. Our approach, composed of a Bayesian formulation together with active exploration behaviours inspired by intrinsic motivation models, overcomes some of the limitations observed in previous works for object recognition, such as constraint to object geometry and size, and predefined sequence and locations of exploration contacts. A detailed description of our method is presented in Section III.

\section{METHODS}

\section{A. Robotic platform}

In this work we use a robotic platform composed of a three-fingered robotic hand and a positioning robotic table (see Figure 1). They are used for systematic data collection and implementation of our proposed method. A detailed description of these robots is presented in the following sections. 


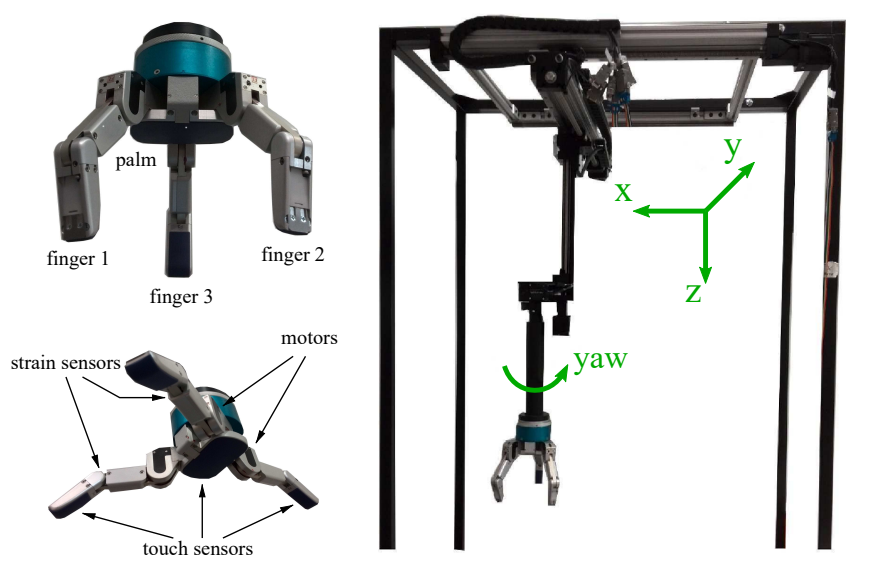

(A)

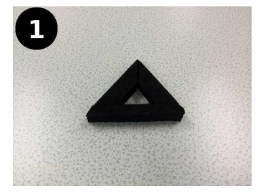

triangle

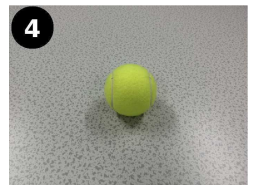

ball 2

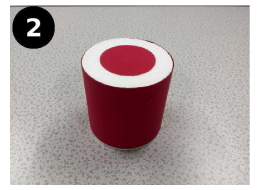

cylinder

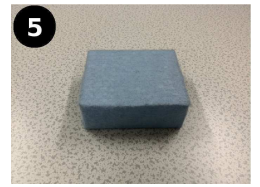

box 1

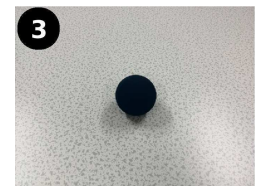

ball 1

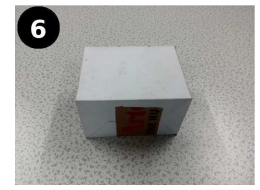

box 2

Fig. 2. Objects used for data collection, training and testing our methods for perception and exploration. Proprioceptive information from the robotic hand was collected during the exploration of each object shape.

Fig. 1. Exploratory robotic platform. (A) Three-fingered robotic hand, composed of 4 degrees of freedom and integrated with tactile and strain sensors. (B) 4 degrees of freedom $(x, y, z, y a w)$ positioning robotic table.

1) Dexterous robotic hand: The three-fingered robotic hand, from Barrett Technology Inc., has 4-DoF; 1-DoF in each finger to perform opening and closing movements, and 1-DoF for spreading the fingers around the palm (see Figure 1A). This robotic hand is integrated with tactile and force sensors. Each finger is composed of 22 taxels (tactile elements), whilst the palm contains 24 taxels of 12 bit resolution each. The strain sensors, located in the base of each finger, permit to detect a contact force to safely stop a finger movement once a force threshold is exceeded. It is also possible to acquire proprioceptive information from the joint angles of the fingers and the spread motor in real-time. The information provided by touch and proprioceptive sensors, available in the robotic hand, is essential for safe and robust robot exploration, perception and interaction with the environment.

2) Positioning robotic table: An exploratory positioning robotic table was built in our laboratory using commercial motors from Newmark Systems Inc. This positioning robotic table, composed of 4-DoF, permits to perform precise exploratory movements in $x-, y-, z-$ axes, and rotations in yaw (see Figure 1B). A controller for precise control and synchronisation of robot movements was designed and implemented in a ChipKit Uno32 board with a PIC microcontroller.

Both robots, the three-fingered robotic hand and the positioning table, were integrated to obtain an 8-DoF exploratory robotic platform. This configuration permits to achieve a large set of exploration movements: 1) opening and closing the fingers; 2) spreading the fingers around the palm; 3) rotation of the wrist (yaw); and 4) displacements in $x-, y-, z-$ axes of the robotic hand in the working space. Furthermore, this robotic platform permits the exploration and manipulation of a large variety of objects by precisely synchronising and controlling the robotic fingers and movements of the positioning table.

For control of robot movements, during data collection and testing of our methods, we developed a set of control and synchronisation modules using $\mathrm{C}++$ programming language and the 'Yet Another Robot Platform' (YARP) library, which has shown its potential for the design and implementation of robust robotic systems in multiple applications.

\section{B. Data collection}

Our work is focused on perception and active exploration behaviours for recognition of object shape using a robotic hand. For this purpose, we collected position and orientation data from the robot hand during the exploration of multiple objects. For data collection we used six objects labelled as triangle, cylinder, ball 1, ball 2, box 1 and box 2 (see Figure 2).

Each object was placed and firmly attached on a fixed base, one at a time, in a target position for robot exploration. The robot hand was rotated in yaw, by the robotic table, following a circular trajectory around the object being explored to collect position and orientation data from the three robotic fingers and wrist (see Figure 3). This process was performed as follows: First, the robotic hand was located in a home position. Second, the hand was moved to the target position for exploration of an unknown object. Third, the robotic fingers started to move to make contact with the object. Then, they stopped as soon as a contact was detected, by exceeding a predefined tactile pressure and force thresholds, to avoid any damage to the hand and object. Next, the fingers were kept in contact with the object for $1 \mathrm{sec}$, giving enough time to collect 50 samples of position and orientation information from the fingers and wrist. Once the data were collected, the fingers were opened to reach again the home position, rotate the wrist and collect data with a new position and orientation of the robotic hand.

A sequence of 30 rotations and tactile contacts around each object was performed with rotations of 12 degrees steps, thereby exploring the complete object. Figure 3 shows an example of the robotic hand exploring two objects at four different orientations. The data collected from each tactile contact for each object were stored in matrices composed of 50 sensor samples (rows) from 5 motors (columns) $(50 \times 5$ matrix); the first three columns are the positions of contacts detected by the finger 1 , finger 2 and finger 3 , the fourth column is the value of the spread motor that controls the separation distance between fingers, and the fifth column is the angle orientation value of the wrist that rotates to perform the complete exploration of each object. The systematic data collection process was repeated ten times per object, forming five datasets for training and five datasets for testing our methods for object exploration and recognition. 


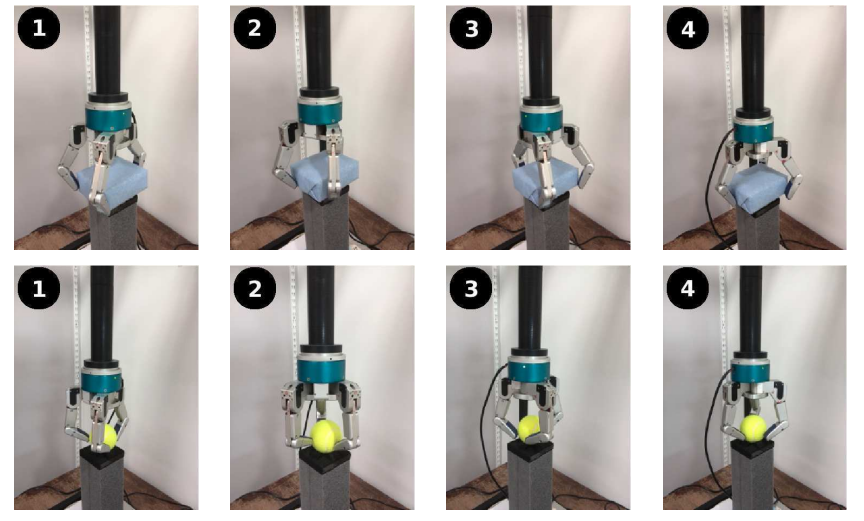

Fig. 3. Data collection through rotations of the robot hand around each object. We show two objects being explored with 4 out of a total of 30 exploration contacts. Position and orientation data are collected during the exploration process to train and test our methods.

\section{Bayesian perception of touch}

Probabilistic models offer a flexible approach for development of robust applications in robotics -for instance, perception and learning through interaction with the environment. In this work, we use a Bayesian formulation that, together with a sequential analysis method, provides an accurate framework for perception and exploration of object shape using position and orientation information from a robotic hand.

Bayesian update: our Bayesian formulation recursively estimates the posterior probabilities from the product of the prior probabilities and likelihoods. Automatic stop of the estimation process, to make a decision about the object being explored, was controlled by a sequential analysis method which uses a belief threshold crossing approach. The formulation of the Bayesian approach is as follows:

$$
P\left(c_{n} \mid z_{t}\right)=\frac{P\left(z_{t} \mid c_{n}\right) P\left(c_{n} \mid z_{t-1}\right)}{P\left(z_{t} \mid z_{t-1}\right)}
$$

where $P\left(c_{n} \mid z_{t}\right)$ is the posterior probability. The prior and likelihood are represented by $P\left(c_{n} \mid z_{t-1}\right)$ and $P\left(z_{t} \mid c_{n}\right)$ respectively. Proper normalised probabilities in $[0,1]$ are obtained with the marginal probability $P\left(z_{t} \mid z_{t-1}\right)$. The object class to be estimated is defined by $c_{n} \in C=1,2, \ldots, N$ with $N=6$, and where each class $c_{n}$ is composed of position and orientation information from the object being explored. The observations from the robotic hand are represented by $z$. The exploration time $t$ is the sequence of contacts performed by the robotic hand on each object. Each component of the Bayesian formulation is detailed in the following paragraphs.

Prior: an initial uniform prior probability is assumed for all the test objects to be explored. The initial prior probability for an object exploration process is defined as follows:

$$
P\left(c_{n}\right)=P\left(c_{n} \mid z_{0}\right)=\frac{1}{N}
$$

where $c_{n}$ is the object class, $z_{0}$ is the observation at time $t=0$ and $N$ is the number of test objects used for exploration.

Measurement model and Likelihood estimation: position and orientation information, from $M_{\text {motors }}$ motors of the robotic platform, are obtained for each contact performed during the object exploration task. In this work, we use $M_{\text {motors }}=5$; four motors from the robotic hand (control of fingers positions) and one motor from the robotic table (control of hand orientation). From each contact we obtain a time series with $N_{\text {samples }}=50$ for each motor. This information is used to obtain the measurement model with a nonparametric estimation method based on histograms. The histograms are uniformly constructed by binning robot contact information into bins $b$ (interval position within a histogram) with $N_{\text {bins }}=100$ intervals. We use these histograms to evaluate a contact $z_{t}$ performed by the robotic hand at time $t$, and estimate the likelihood of a perceptual class $c_{n} \in C$. The measurement model is formulated as follows:

$$
P_{m}\left(b \mid c_{n}\right)=\frac{h_{m, n}(b)}{\sum_{b=1}^{N_{\text {bins }}} h(b)}
$$

where $h_{m, n}(b)$ is the sample count in bin $b$ for motor $m$ over all training data in class $c_{n}$. The values are normalised by $\sum_{b=1}^{N_{\text {bins }}} h(b)$ to have proper probabilities that sum to 1 . Thus, the likelihood of the contact $z_{t}$ at time $t$ by evaluating Equation (3) over all motors and samples is as follows:

$$
\log P\left(z_{t} \mid c_{n}\right)=\sum_{m=1}^{M_{\text {motors }}} \sum_{j=1}^{N_{\text {samples }}} \frac{\log P_{m}\left(s_{m}(j) \mid c_{n}\right)}{M_{\text {motors }} N_{\text {samples }}}
$$

where $s_{m}(j)$ is the sample $j$ in motor $m$, and $P\left(z_{t} \mid c_{n}\right)$ is the likelihood of the measurement $z_{t}$ given a perceptual class $c_{n}$. Properly normalised values are ensured with the marginal probabilities conditioned from the previous contact as follows:

$$
P\left(z_{t} \mid z_{t-1}\right)=\sum_{n=1}^{N} P\left(z_{t} \mid c_{n}\right) P\left(c_{n} \mid z_{t-1}\right)
$$

Stop decision for object recognition: the accumulation of evidence with the Bayesian update process stops once a belief threshold is exceeded, making a decision about the object being explored. Thus, the object perceptual class is obtained using the maximum a posteriori (MAP) estimate as follows:

$$
\begin{gathered}
\text { if any } P\left(c_{n} \mid z_{t}\right)>\beta_{\text {threshold }} \text { then } \\
\hat{c}=\underset{c_{n}}{\arg \max } P\left(c_{n} \mid z_{t}\right)
\end{gathered}
$$

where the object estimated at time $t$ is represented by $\hat{c}$. The belief threshold $\beta_{\text {threshold }}$ permits to adjust the confidence level for the decision making process. We used a set of belief thresholds $\beta_{\text {threshold }}=\{0.0,0.05, \ldots, 0.99\}$ to observe their effects on the accuracy of the object recognition process.

\section{Active exploration behaviours}

We integrated our Bayesian formulation for perception together with active exploration behaviours. These behaviours allow to observe the effects, in perception accuracy and reaction time, when the robot hand is purposefully guided to explore interesting object locations that improve perception. We also implement a passive exploration behaviour to compare its performance with our proposed active exploration methods. 
1) passive exploration: Passive exploration is defined in this work as the exploration of object locations that are not purposefully selected, but instead, they are randomly chosen from a uniform probability distribution. These locations, defined as sensorimotor states (SM), represent the angle orientation for exploration of specific object locations. The passive behaviour for object exploration is perform as follows:

$$
a_{\text {passive }}=\operatorname{Random}(\operatorname{SM}(t))
$$

where $a_{\text {passive }}$ is the action chosen, from the random selection of the SM states at exploration time $t$, to control the movements of the robot hand.

2) active exploration: For the active exploration, we developed two novel computational methods; familiarity and novelty exploration behaviours. These methods are inspired by the intrinsic motivation approach, which has shown to be primordial to engage humans to explore, interact and manipulate their surrounding environment [12], [13].

The familiarity and novelty behaviours allow to actively control robot movements, for exploration of better locations that improve object perception [48], [49]. The familiarity behaviour actively seeks object locations that provide low levels of interestingness, while the novelty model guides the exploration towards object locations with high levels of interestingness. Here, we propose an interestingness method for intrinsic motivation that, inspired by the predictive knowledgebased motivation models [44], [45], predicts the sensorimotor states (SM) for future events, based on the product of past and current prediction errors as follows:

$$
\mathrm{I}(\mathrm{SM}(t))=e_{\mathrm{I}}(t-1) \cdot e_{\mathrm{I}}(t)
$$

where $\mathrm{I}(\operatorname{SM}(t))$ is the level of interestingness for the sensorimotor states $\mathrm{SM}$ at time $t$, while $e_{\mathrm{I}}(t)$ and $e_{\mathrm{I}}(t-1)$ are the prediction errors from current and past times $t$ and $t-1$. From Equation (8), it comes naturally to use the lowest and highest prediction errors to model intrinsic motivation for familiarity and novelty behaviours [13], [43]. The prediction error $e_{\mathrm{I}}(t)$ is defined as the distance between the posterior from the Bayesian update, which contains the probability of each orientation for each object class at time $t$, and the belief threshold used to make a decision as follows:

$$
e_{\mathrm{I}}(t)=P\left(c_{n} \mid z_{t}\right)-\beta_{\text {threshold }}
$$

From Equations (8) and (9) it is possible to define exploration behaviours to actively control the robot hand, based on the selection of either SM states with low (familiarity) or high (novelty) levels of interestingness as follows:

$$
\begin{gathered}
a_{\text {familiarity }}=\underset{\text { SM }}{\arg \min } \mathrm{I}(\mathrm{SM}(t)) \\
a_{\text {novelty }}=\underset{\mathrm{SM}}{\arg \max } \mathrm{I}(\mathrm{SM}(t))
\end{gathered}
$$

where $a_{\text {familiarity }}$ and $a_{\text {novelty }}$ are the selected robot actions with low and high levels of interestingness for object exploration. This approach, together with the Bayesian formulation, is repeated until the belief threshold used to make a decision about the object being explored is exceeded. This

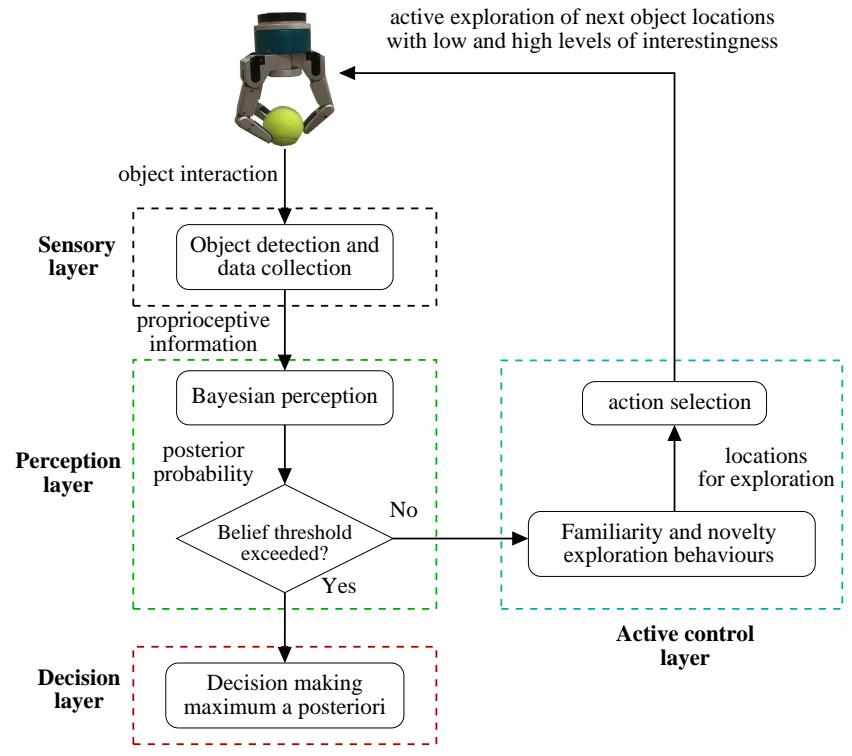

Fig. 4. Flowchart for active object exploration using probabilistic perception and intrinsic motivation. The three-fingered robot hand is actively controlled to explore locations with low (familiarity) and high (novelty) interestingness levels to improve perception. The exploration and perception processes are repeated until a predefined belief threshold is exceeded, to make a decision about the object being explored.

process is described in the flowchart of Figure 4, composed of four layers; sensory, perception, decision and active control.

\section{RESUlts}

For validation of our methods, various experiment for object exploration and recognition were implemented in both off-line and real-time modes. In off-line mode we used the datasets obtained from the data collection process, while in real-time mode we used the robotic platform described in Section III-A. These experiments are described in the following sections.

\section{A. Off-line object exploration}

We implemented an object exploration and recognition task in off-line mode using active and passive exploration behaviours. For training and testing the proposed methods, we employed ten datasets (five for training and five for testing) obtained from the data collection process in Section III-B.

1) Passive object exploration: For object recognition with passive exploration behaviour, the Bayesian formulation performed a random selection of object locations for exploration. In this experiment, data collected from objects in Figure 2 were used. The decisions made by the perception method were controlled by the belief thresholds $\beta_{\text {threshold }}=\{0.0,0.05, \ldots$, $0.99\}$, to observe their effects in accuracy and reaction time. To ensure robustness in results, the recognition task was repeated 10,000 times for each belief threshold value, and randomly selecting the initial location for exploration.

The red colour curve in Figure 5A shows the recognition accuracy for passive exploration, where the solid and dashed lines are the mean and standard deviation over all test objects. We observe that the mean error decreased for large belief thresholds, which shows that accumulation of evidence allows 


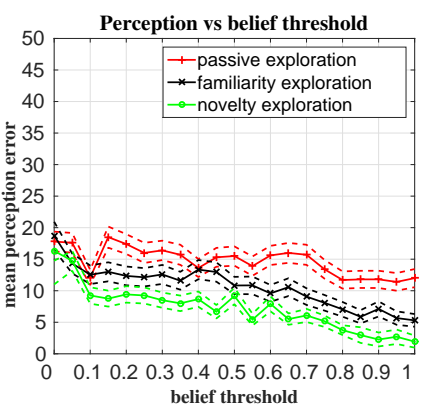

(A)

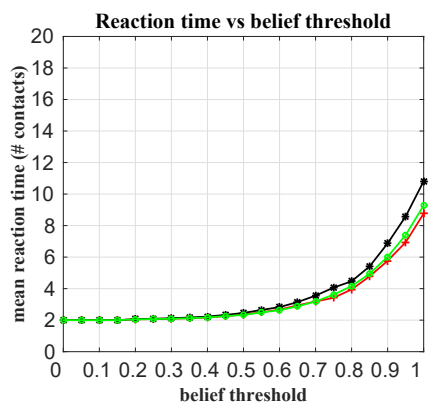

(B)
Fig. 5. Mean (solid lines) and standard deviation (dashed lines) results from the exploration of all test objects in off-line mode. (A) Perception against belief threshold for passive and active exploration, where the latter implements the familiarity and novelty behaviours to explore locations with low and high levels of interestingness. The highest recognition accuracy is achieved by the novelty behaviour. (B) Reaction time was not drastically affected, showing similar results for both passive and active exploration behaviours.

to improve perception. However, the smallest error of $12.5 \%$ obtained with the highest belief threshold is large compared to the results achieved by active exploration behaviours described in next paragraphs. The reaction time or number of contacts required to make a decision, gradually increased to a maximum of 9 contacts per decision for large belief thresholds as shown in Figure 5B (red colour curve). These results were obtained by averaging all perceptual classes for each belief threshold.

2) Active object exploration: For active exploration, we implemented an object recognition task with both familiarity and novelty exploration behaviours. The objective is to control the robot exploration movements towards object locations with different levels of interestingess to improve perception. To ensure robustness of the experiment, the task was repeated 10,000 times for each belief threshold in $\beta_{\text {threshold. Fur- }}$ thermore, the initial location for exploration was randomly selected, and then the exploration was controlled by the familiarity and novelty behaviours.

The familiarity exploration behaviour was implemented using the Bayesian formulation together with the exploration strategy described by Equations (8),(9),(10). This approach actively selects familiar locations for exploration that represent low levels of interestingness. Black colour curves in Figure 5A show the mean (solid line) and standard deviation (dashed line) results. A decreasing perception error for large belief thresholds is observed, achieving the smallest error of $5 \%$ with $\beta_{\text {threshold }}=0.99$. The results show an improvement over the random selection (passive exploration) of object locations. However, the reaction time was affected by the familiarity behaviour, requiring a larger number of contacts to make a decision (see black colour curve in Figures 5B).

The object recognition task was repeated using the novelty exploration behaviour. This approach, described by Equations (8),(9),(11), selects object locations that contain high levels of interestingness for exploration. The mean (solid line) and standard deviation (dashed line) results are shown by the green colour curves in Figure 5A. These results present the improvement in perception accuracy, achieving the smallest error of $2 \%$ with $\beta_{\text {threshold }}=0.99$. It is important to observe that the reaction time was not largely affected compared to the results from the familiarity behaviour. A maximum of 9 sensor contacts were required to make a decision for the highest perception accuracy (green colour curve in Figure 5B). These results show that exploring high interesting locations not only improve the recognition performance, but also provide a better exploration and exploitation trade-off, ensuring high accuracy without significantly affecting the decision making time.

\section{B. Real-time object exploration}

Validation in real-time mode with an object recognition task using the robot platform described in Section III-A. In these experiments, we used the test objects shown in Figure 2.

1) Passive object exploration: For object recognition with passive exploration behaviour, the robot movements were controlled by random selection of locations for exploration around the object. All the test objects were placed on a table, one at a time, for exploration and recognition using the following process. First, the robot hand was moved from its home position to a predefined location to start the object exploration. Second, the robot made a contact on the object to build an initial belief of the object being explored. Then, the posterior probability was estimated using our Bayesian formulation. This process was repeated by the robot, selecting random object locations and accumulating evidence from the object. The exploration task was stopped once the updated posterior probability exceeded a belief threshold, allowing the robot to make a decision and recognition of the explored object. This experiment was performed using the belief thresholds $\beta_{\text {threshold }}=\{0.0,0.05, \ldots, 0.99\}$ to control the accumulation of evidence and evaluate the effects on perception accuracy and reaction time.

Perception accuracy results are shown by the black colour bars in Figure 6A for three different belief thresholds. We observe that the object recognition error, for the task described in the previous paragraph, is gradually improved with $28.50 \%$, $18.30 \%$ and $10.0 \%$ errors for belief thresholds of $0.0,0.5$ and 0.99 respectively. The corresponding reaction times to make a decision are shown in Figure 6B. This result shows that 2 and 15 tactile contacts were employed by the robot hand to achieve the largest and smallest perception errors of $28.50 \%$ and $10 \%$. The recognition accuracy for each object using $\beta_{\text {threshold }}=$ 0.99 is presented by the confusion matrix in Figure 6C.

2) Active object exploration: The object recognition task was implemented using the familiarity and novelty behaviours. These approaches actively explored an object by intelligent robot movements towards object locations that represent low and high levels of interestingness. Active exploration differs from passive exploration, described in the previous paragraph, in the method for selection of object locations for exploration.

The familiarity exploration behaviour allowed the robot hand to estimate the object locations with low levels of interestingness. These locations were used to actively control the movements of the robot for the object exploration task. The exploration process was repeated until the belief threshold value $\beta_{\text {threshold }}=\{0.0,0.05, \ldots, 0.99\}$ was exceeded by the posterior probability. The novelty exploration behaviour allowed the active controlled of the robot hand movements towards object locations with high levels of interestingness. 
Similar to the familiarity model, the posterior probability was recursively updated until the belief threshold was exceeded, and then, making a decision about the object being explored. These experiments with both familiarity and novelty exploration behaviours were performed in real-time mode using the test objects shown in Figure 2 and three belief thresholds $(0.0,0.5$, and 0.99).

Perception accuracy results for object recognition using the familiarity and novelty exploration behaviours are shown by the grey and white colour bars in Figure 6A. We observe that perception with the familiarity approach achieved errors of $24 \%, 12.2 \%$ and $4 \%$ for belief thresholds of $0.0,0.5$ and 0.99 . These values were improved by the novelty approach which achieved errors of $13.3 \%, 9.8 \%$ and $1 \%$. On the one hand, both active exploration behaviours were able to obtain better perception accuracy over the passive approach. On the other hand, the familiarity and novelty behaviours slightly increased the reaction time with a mean of 16 sensor contacts needed for the highest perception accuracy (see Figure 6B).

The recognition accuracy for individual objects using active exploration behaviours is presented by the confusion matrices in Figures 6D,E. The smallest error in object recognition was achieved by the novelty exploration behaviour, whilst the passive approach obtained the largest error. The blue colour region around each object shown in Figure 7 shows the locations explored by the robot hand. These regions represent the object locations with low and high levels of interestingness, estimated by the familiarity and novelty behaviours. The results from all the experiments presented in this section show the benefits of actively controlling the robot hand towards interesting locations for exploration. Furthermore, we observed that exploration of high interesting locations, with the novelty behaviour, allowed to achieve higher accuracy for object recognition in both off-line and real-time modes.

\section{DISCUSSION}

An investigation of novel perception and exploration methods for object recognition with a robotic hand was presented in this work. First, we demonstrated how probabilistic perception methods benefit object recognition tasks by the accumulation of evidence and dealing with uncertainty present in the environment. Second, we showed that integration of familiarity and novelty behaviours, for active exploration of interesting object locations, provide a better trade-off between perception accuracy and decision making time over passive exploration.

Probabilistic Bayesian perception allowed the robotic hand to reduce uncertainty from sensor measurements, through the iterative interaction with the object being explored. This approach, together with a sequential analysis method based on threshold crossing, permitted the robot to autonomously decide whether or not the accumulated evidence was enough to make a decision. The sequential analysis method was implemented with a set of belief thresholds, which allowed to observe that large amounts of evidence, intelligently accumulated during the exploration task and controlled by large belief threshold values, provide a gradual improvement in perception accuracy.

Passive and active exploration behaviours were implemented, together with the probabilistic perception approach, to control robot movements to explore object locations that represent different levels of interestingness. Passive exploration, based on random selection of object locations, showed a slight improvement in accuracy for large belief thresholds, achieving errors of $12.5 \%$ and $10 \%$ with experiments in off-line and real-time modes. This approach did not use any systematic movement or intelligent decision control to decide where to move next to explore better object locations for perception. A gradual increment in the reaction time was observed for large belief thresholds. This result was expected given that for large threshold values more evidence is needed to make a decision.

Active exploration for object recognition was studied with two approaches; familiarity and novelty behaviours. These behaviours, inspired by the intrinsic motivation approach, were used to control robot movements to intelligently explore object locations that represent low (familiar) and high (novel) levels of interestingness. Here, we defined familiarity as the locations with low levels of interestingness, in other words, locations that do not provide new information to improve perception (see Section III-D). The accuracy of the object recognition task, using the familiarity behaviour, was gradually improved in both off-line and real-time modes, achieving small perception errors of $5 \%$ and $4 \%$ respectively with the belief threshold of 0.99 . An expected increment in the reaction time was also observed for large belief thresholds, increasing the number of

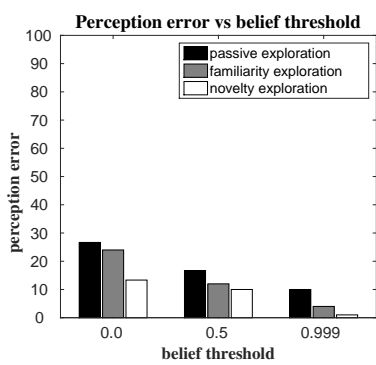

(A)

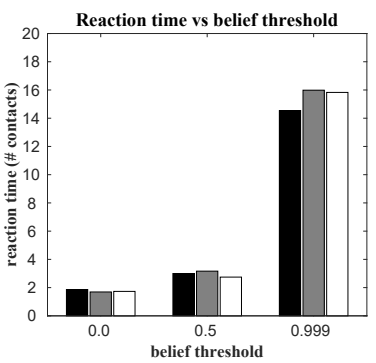

(B)

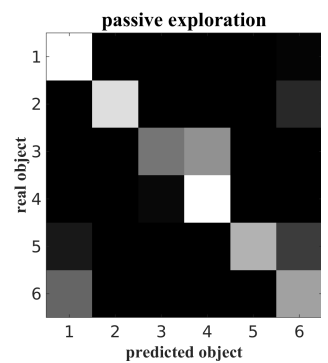

(C)

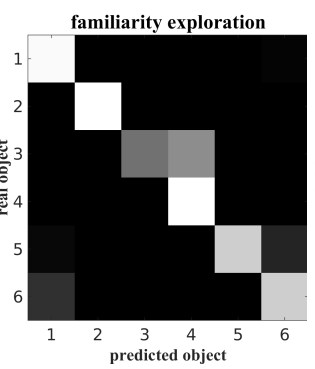

(D)

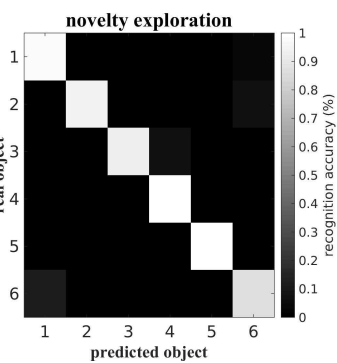

(E)

Fig. 6. Perception and reaction time results, averaged over all test objects from real world experiments for passive and active exploration behaviours. (A) Perception error decreased, achieving small values, for active exploration behaviour. Exploration of locations with high (novelty) levels of interestingness achieved the best perception. (B) Reaction time increased for larger values of belief threshold. Similar reaction times were observed for both passive and active exploration. Confusion matrices from object recognition results with passive (C) and active (D, E) exploration. The test objects used for the experiments are: 1) triangle, 2) cylinder, 3) blue 1,4) ball 2, 5) box 1 and 6) box 2. Even though improvements were achieved by all the exploration behaviours, the best performance was obtained with the novelty approach, which explored the locations with high levels of interestingness. 


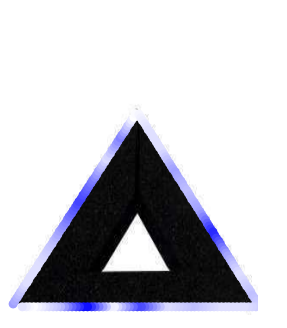

triangle

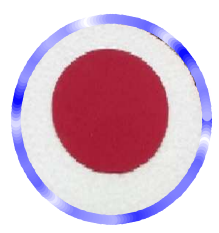

cylinder

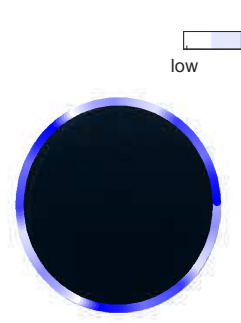

ball 1
Interestingness

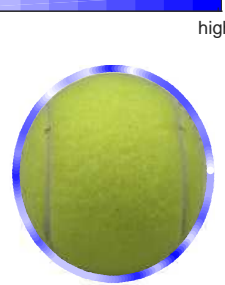

ball 2

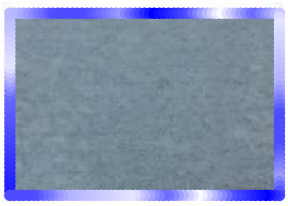

box 1

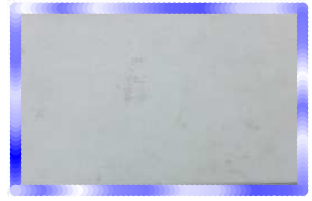

box 2

Fig. 7. Object locations with low and high levels of interestingness estimated from exploration experiments, using our Bayesian formulation for perception together with familiarity and novelty intrinsic motivation models. Levels of interestingness around objects are represented by the blue colour bar. Dark-blue colour locations provided better stimuli to improve perception accuracy over locations with white and light-blue colours.

sensor contacts needed to make a decision. Even though the reaction time was affected, it did not change drastically with respect to the results from the passive exploration behaviour.

The novelty exploration behaviour, in contrast to the familiarity approach, controlled the robot hand to explore object locations that represent high levels of interestingness. These locations, estimated with the formulation in Section III-D, provide novel information useful to improve both perception accuracy and reaction time. High interesting object locations could be those unknown or novel locations that have not been explored yet. On the one hand, these locations initially present high uncertainty, but on the other hand, they also offer a rich source of information with the potential to help the robot to recognise, more accurately and quickly, the objects being explored. Results showed that intelligently exploring object locations with high levels of interestingness, allows the robot hand to achieve the smallest perception errors of $2 \%$ and $1 \%$ for large belief threshold with experiments in off-line and realtime modes respectively. Similar to previous experiments, the reaction time was gradually increased, however, the number of contacts needed to make a decision was smaller than the number required by the familiarity exploration behaviour.

From the experiments we observed that, on the one hand, active exploration improved the accuracy for object recognition over passive exploration. On the other hand, the novelty behaviour is the active exploration method that achieved the best perception accuracy without largely affecting the reaction time. First, these results suggest that passive exploration (random selection of object locations) does not allow to perform a systematic accumulation of evidence, which affects the performance in accuracy and reaction time of our probabilistic perception approach. Second, exploration of familiar locations only (locations with low levels of interestingness based on the familiarity behaviour), does not provide new information after some time, making the accumulation of evidence a redundant process. Therefore, intelligent control of robot interaction with object locations that contain novel or high interesting stimuli, offers a better exploration and exploitation trade-off for perception accuracy and reaction time. Object recognition results from all the exploration approaches are presented by the confusion matrices in Figures $6 \mathrm{C}, \mathrm{D}, \mathrm{E}$, where we observe that the best recognition accuracy is achieved by the novelty model. It is important to note that actively moving the robot hand provides multiple benefits not only over the passive approach, but also, over a complete and predefined sequence of exploration contacts, e.g., exploration of all the positions used for data collection. These benefits offered by the active approach are: 1) intelligent movement decisions based on intrinsic motivation, which is primordial to engage an agent to explore its environment, 2) small number of sensor samples required to make a decision, 3) exploration of object locations that improve perception to achieve high recognition accuracy, and 4) active robot movements towards interesting object locations that mimic the way in that humans explore objects in their environment.

The low and high levels of interestingness estimated around each object, employed in the recognition experiments, are represented by light and dark-blue colour regions in Figure 7. These regions show the result of actively controlling the robotic hand during the object exploration task. The darkblue colour regions show the explored locations that, based on the novelty behaviour, provided better information to improve object recognition. This exploration process mimics the way in that humans actively move their hands and fingers, looking for better or interesting information, to reduce uncertainty and successfully recognise multiple objects.

Our proposed methods for object exploration and recognition using intrinsic motivation models demonstrated to be accurate and fast, intelligently moving a robot hand around an object. However, our method is currently limited to the use of touch only to detect an object contact, without contributing to the object recognition process. Also, the robot hand pose (position and orientation) is constrained to perform a contourbased exploration approach. All these challenging aspects are planned to be investigated in future work.

Exploration and recognition tasks with robot hands not only need to touch, but also to feel and search for interesting information. For that reason, in this work we offered a robust framework for perception and exploration to achieve autonomous and intelligent robots capable to touch, feel and recognise objects located in their surrounding environment.

\section{CONCLusion}

In this work, we demonstrated for the first time that probabilistic perception methods, together with active exploration behaviours, allow a robotic hand to successfully explore and recognise objects. This process is achieved by actively controlling the exploration movements towards interesting object locations to improve the perception about the object being explored. We also showed how actively exploring an 
object improves the reaction time over a passive exploration approach. Our methods were validated with object recognition experiments in both off-line and real-time modes using an exploratory robot platform. The results showed that our approach has the potential to make a robotic hand capable to touch, perceive and autonomously decide where to move next, to extract better information in exploration and recognition tasks.

\section{REFERENCES}

[1] U. Martinez-Hernandez, "Tactile sensors," in Scholarpedia of Touch. Springer, 2016, pp. 783-796.

[2] J. Dargahi and S. Najarian, "Advances in tactile sensors design/manufacturing and its impact on robotics applications-a review," Industrial Robot: An International Journal, vol. 32, no. 3, pp. 268-281, 2005.

[3] U. Martinez-Hernandez, L. Boorman, and T. Prescott, "Multisensory wearable interface for immersion and telepresence in robotics," IEEE Sensors Journal, vol. 17, no. 8, pp. 2534-2541, 2017.

[4] T. J. Prescott, M. E. Diamond, and A. M. Wing, "Active touch sensing," Phil. Trans. R. Soc. B, vol. 366, pp. 2989-2995, 2011.

[5] S. Lederman and R. Klatzky, "Haptic perception: A tutorial," Attention, Perception, and Psychophysics, vol. 71, pp. 1439-1459, 2009.

[6] U. Martinez-Hernandez, T. J. Dodd, M. H. Evans, T. J. Prescott, and N. F. Lepora, "Active sensorimotor control for tactile exploration," Robotics and Autonomous Systems, vol. 87, pp. 15-27, 2017.

[7] U. Martinez-Hernandez, I. Mahmood, and A. A. Dehghani-Sanij, "Probabilistic locomotion mode recognition with wearable sensors," in Converging Clinical and Engineering Research on Neurorehabilitation II. Springer, 2017, pp. 1037-1042.

[8] U. Martinez-Hernandez, A. Rubio-Solis, and T. J. Prescott, "Bayesian perception of touch for control of robot emotion," in Neural Networks (IJCNN), 2016 International Joint Conference on. IEEE, 2016, pp. 4927-4933.

[9] S. Thrun, W. Burgard, and D. Fox, Probabilistic robotics. MIT press, 2005.

[10] S. Thrun, "Probabilistic algorithms in robotics," AI Magazine, vol. 21, no. 4, p. 93, 2000.

[11] U. Martinez-Hernandez, N. F. Lepora, and T. J. Prescott, "Active haptic shape recognition by intrinsic motivation with a robot hand," in World Haptics Conference (WHC), 2015 IEEE. IEEE, 2015, pp. 299-304.

[12] R. M. Ryan and E. L. Deci, "Intrinsic and extrinsic motivations: Classic definitions and new directions," Contemporary educational psychology, vol. 25 , no. 1, pp. 54-67, 2000.

[13] A. G. Barto, S. Singh, and N. Chentanez, "Intrinsically motivated learning of hierarchical collections of skills," in Proc. 3rd Int. Conf. Development Learn, 2004, pp. 112-119.

[14] F. Kaplan and P.-Y. Oudeyer, "Intrinsically motivated machines," 50 years of artificial intelligence, pp. 303-314, 2007.

[15] S. Lederman and R. Klatzky, "Extracting object properties through haptic exploration," Acta Psychologica, vol. 84, no. 1, pp. 29 - 40, 1993.

[16] T. Prescott, M. Pearson, B. Mitchinson, J. Sullivan, and A. Pipe, "Whisking with robots," Robotics Automation Magazine, IEEE, vol. 16, no. 3, pp. 42-50, 2009

[17] S. Stansfield, "Primitives, features, and exploratory procedures: Building a robot tactile perception system," in Robotics and Automation. Proceedings. 1986 IEEE International Conference on, vol. 3. IEEE, 1986, pp. 1274-1279.

[18] S. A. Stansfield, "Haptic perception with an articulated, sensate robot hand," Robotica, vol. 10, no. 06, pp. 497-508, 1992.

[19] J. W. Roach, P. K. Paripati, and M. Wade, "Model-based object recognition using a large-field passive tactile sensor,' IEEE Transactions on Systems, Man, and Cybernetics, vol. 19, no. 4, pp. 846-853, 1989.

[20] V. Riffo and D. Mery, "Automated detection of threat objects using adapted implicit shape model," IEEE Transactions on Systems, Man, and Cybernetics: Systems, vol. 46, no. 4, pp. 472-482, 2016.

[21] A. Jimenez, A. Soembagijo, D. Reynaerts, H. Van Brussel, R. Ceres, and J. Pons, "Featureless classification of tactile contacts in a gripper using neural networks," Sensors and Actuators A: Physical, vol. 62, no. 1, pp. 488-491, 1997.

[22] A. M. Okamura, M. L. Turner, and M. R. Cutkosky, "Haptic exploration of objects with rolling and sliding," in Robotics and Automation, 1997. Proceedings., 1997 IEEE International Conference on, vol. 3. IEEE, 1997, pp. 2485-2490.
[23] N. Gorges, S. E. Navarro, D. Goger, and H. Worn, "Haptic object recognition using passive joints and haptic key features," in Robotics and Automation (ICRA), 2010 IEEE International Conference on. IEEE, 2010, pp. 2349-2355.

[24] S. Ratnasingam and T. M. McGinnity, "Object recognition based on tactile form perception," in Robotic Intelligence In Informationally Structured Space (RiiSS), 2011 IEEE Workshop on. IEEE, 2011, pp. 26-31.

[25] K. S. Roberts, "Robot active touch exploration: Constraints and strategies," in Robotics and Automation, 1990. Proceedings., 1990 IEEE International Conference on. IEEE, 1990, pp. 980-985.

[26] J. S. Bay, "A fully autonomous active sensor-based exploration concept for shape-sensing robots," IEEE Transactions on Systems, Man, and Cybernetics, vol. 21, no. 4, pp. 850-860, 1991.

[27] M. Johnsson and C. Balkenius, "Neural network models of haptic shape perception," Robotics and Autonomous Systems, vol. 55, no. 9, pp. 720727, 2007.

[28] H. Liu, J. Greco, X. Song, J. Bimbo, L. Seneviratne, and K. Althoefer, "Tactile image based contact shape recognition using neural network," in Multisensor Fusion and Integration for Intelligent Systems (MFI), 2012 IEEE Conference on. IEEE, 2012, pp. 138-143.

[29] F. Sun, C. Liu, W. Huang, and J. Zhang, "Object classification and grasp planning using visual and tactile sensing," IEEE Transactions on Systems, Man, and Cybernetics: Systems, vol. 46, no. 7, pp. 969-979, 2016.

[30] U. Martinez-Hernandez and T. J. Prescott, "Expressive touch: Control of robot emotional expression by touch," in Robot and Human Interactive Communication (RO-MAN), 2016 25th IEEE International Symposium on. IEEE, 2016, pp. 974-979.

[31] D. Codetta-Raiteri and L. Portinale, "Dynamic bayesian networks for fault detection, identification, and recovery in autonomous spacecraft," IEEE Transactions on Systems, Man, and Cybernetics: Systems, vol. 45, no. 1, pp. 13-24, 2015.

[32] M. Meier, M. Schöpfer, R. Haschke, and H. Ritter, "A probabilistic approach to tactile shape reconstruction," Robotics, IEEE Transactions on, vol. 27, no. 3, pp. 630-635, 2011.

[33] N. Gorges, S. E. Navarro, and H. Wörn, "Haptic object recognition using statistical point cloud features," in Advanced Robotics (ICAR), 2011 15th International Conference on. IEEE, 2011, pp. 15-20.

[34] A. Schneider, J. Sturm, C. Stachniss, M. Reisert, H. Burkhardt, and W. Burgard, "Object identification with tactile sensors using bag-offeatures," in Intelligent Robots and Systems, 2009. IROS 2009. IEEE/RSJ International Conference on. IEEE, 2009, pp. 243-248.

[35] U. Martinez-Hernandez, A. Damianou, D. Camilleri, L. W. Boorman, N. Lawrence, and T. J. Prescott, "An integrated probabilistic framework for robot perception, learning and memory," in Robotics and Biomimetics (ROBIO), 2016 IEEE International Conference on. IEEE, 2016, pp. 1796-1801.

[36] H. Soh and Y. Demiris, "Incrementally learning objects by touch: Online discriminative and generative models for tactile-based recognition," Haptics, IEEE Transactions on, vol. 7, no. 4, pp. 512-525, 2014.

[37] U. Martinez-Hernandez, T. Dodd, T. J. Prescott, and N. F. Lepora, "Active bayesian perception for angle and position discrimination with a biomimetic fingertip," in Intelligent Robots and Systems (IROS), 2013 IEEE/RSJ International Conference on. IEEE, 2013, pp. 5968-5973.

[38] U. Martinez-Hernandez, T. J. Dodd, L. Natale, G. Metta, T. J. Prescott, and N. F. Lepora, "Active contour following to explore object shape with robot touch," in World Haptics Conference (WHC), 2013. IEEE, 2013, pp. 341-346.

[39] A. Borji, D. N. Sihite, and L. Itti, "What/where to look next? modeling top-down visual attention in complex interactive environments," IEEE Transactions on Systems, Man, and Cybernetics: Systems, vol. 44, no. 5, pp. 523-538, 2014.

[40] A. Stout, G. D. Konidaris, and A. G. Barto, "Intrinsically motivated reinforcement learning: A promising framework for developmental robot learning," DTIC Document, Tech. Rep., 2005.

[41] G. Baldassarre and M. Mirolli, "Intrinsically motivated learning systems: an overview," in Intrinsically motivated learning in natural and artificial systems. Springer, 2013, pp. 1-14.

[42] X. Huang and J. Weng, "Novelty and reinforcement learning in the value system of developmental robots," pp. 47-55, 2002.

[43] P.-Y. Oudeyer and F. Kaplan, "What is intrinsic motivation? a typology of computational approaches," Frontiers in neurorobotics, vol. 1, 2007.

[44] P.-Y. Oudeyer, F. Kaplan, and V. V. Hafner, "Intrinsic motivation systems for autonomous mental development," Evolutionary Computation, IEEE Transactions on, vol. 11, no. 2, pp. 265-286, 2007. 
[45] J. Gottlieb, P.-Y. Oudeyer, M. Lopes, and A. Baranes, "Informationseeking, curiosity, and attention: computational and neural mechanisms," Trends in cognitive sciences, vol. 17, no. 11, pp. 585-593, 2013.

[46] H. Q. Ngo, M. D. Luciw, A. Foerster, and J. Schmidhuber, "Learning skills from play: Artificial curiosity on a katana robot arm." in IJCNN, 2012, pp. 1-8.

[47] L. Pape, C. M. Oddo, M. Controzzi, C. Cipriani, A. Förster, M. C. Carrozza, and J. Schmidhuber, "Learning tactile skills through curious exploration," Frontiers in Neurorobotics, vol. 6, 2012.

[48] A. Baranes and P.-Y. Oudeyer, "Active learning of inverse models with intrinsically motivated goal exploration in robots," Robotics and Autonomous Systems, vol. 61, no. 1, pp. 49-73, 2013.

[49] - "Intrinsically motivated goal exploration for active motor learning in robots: A case study," in Intelligent Robots and Systems (IROS), 2010 IEEE/RSJ International Conference on. IEEE, 2010, pp. 1766-1773.

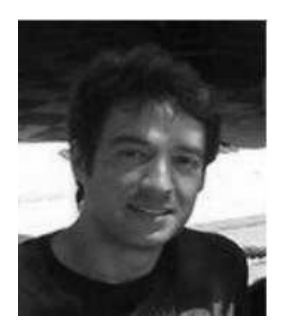

Uriel Martinez-Hernandez received the BEng degree in Communications and Electronics from the National Polytechnic Institute, Mexico City, Mexico, the MSc degree in Computer Sciences from the Centre for Research and Advanced Studies, Mexico City, Mexico, and the $\mathrm{PhD}$ degree from the Department of Automatic Control and Systems Engineering, University of Sheffield, Sheffield, U.K., in 2014.

He was previously a Research Associate at the Sheffield Robotics Laboratory and the Department of Psychology, University of Sheffield. He is currently a Research Fellow at the Institute of Design, Robotics and Optimisation (iDRO) and the School of Mechanical Engineering, University of Leeds. His research interests include machine learning, autonomous robotics, robot perception and control, haptics, wearable and assistive robotics.

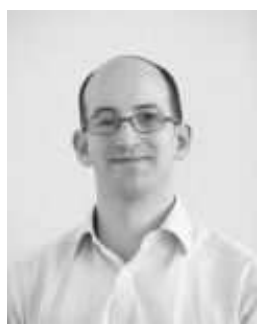

Tony J. Dodd received the BEng in Aerospace Systems Engineering and the $\mathrm{PhD}$ degree from the Department of Electronics and Computer Science at the University of Southampton, UK.

He was previously a research associate at the University of Southampton and University of Sheffield. He became the Director of Undergraduate Studies for Aerospace Engineering in 2009 and was promoted to Senior Lecturer in 2010 . He is currently a Professor of Autonomous Systems Engineering in the Department of Automatic Control \& Systems Engineering, The University of Sheffield, UK. In 2015 he became Director of the MEng degree program in the Faculty of Engineering. His research interests include autonomous vehicles and robotics with particular interest on machine learning, systems engineering, control, manipulation, co-operation and swarming, mission management and optimisation.

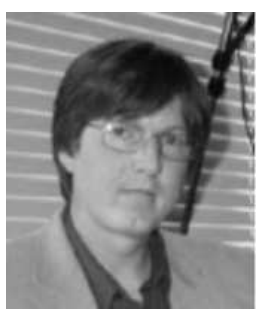

Tony J. Prescott received the M.A. degree in psychology from University of Edinburgh, Edinburgh, U.K., the M.Sc. degree in Artificial Intelligence from the University of Aberdeen, Aberdeen, U.K., and the Ph.D. degree from the Department of Psychology, University of Sheffield, Sheffield, U.K., in 1994.

$\mathrm{He}$ is a currently a Professor of cognitive neuroscience with the Department of Psychology, University of Sheffield. He is also a Permanent Research Fellow with the Bristol Robotics Laboratory, Bristol, U.K. He has authored more than 60 publications in psychology, neuroscience, computational modelling and robotics. His research interests include the biological and brain sciences, particularly concerned with understanding the evolution, development, and function of natural intelligence and the investigation of computational neuroscience models of animal and human intelligence and in testing these models in biomimetic robots. 